OPEN ACCESS

Edited by:

Ragnar Huhn, University Hospital of Düsseldorf, Germany

Reviewed by:

Martin Ströthoff, University Hospital of Düsseldorf, Germany

Claudia Penna

University of Turin, Italy

${ }^{*}$ Correspondence: Alberto Domínguez-Rodríguez adrvdg@hotmail.com

tThese authors share first authorship

Specialty section: This article was submitted to Cardiovascular Therapeutics, a section of the journal Frontiers in Cardiovascular Medicine

Received: 15 December 2020 Accepted: 06 April 2021

Published: 12 May 2021

Citation

Domínguez-Rodríguez A, Abreu-González P, Báez-Ferrer N,

Reiter RJ, Avanzas $P$ and

Hernández-Vaquero D (2021) Melatonin and Cardioprotection in Humans: A Systematic Review and

Meta-Analysis of Randomized Controlled Trials.

Front. Cardiovasc. Med. 8:635083. doi: 10.3389/fcvm.2021.635083

\section{Melatonin and Cardioprotection in Humans: A Systematic Review and Meta-Analysis of Randomized Controlled Trials}

\author{
Alberto Domínguez-Rodríguez ${ }^{1,2 * t}$, Pedro Abreu-González ${ }^{3}$, Néstor Báez-Ferrer ${ }^{1}$, \\ Russel J. Reiter ${ }^{4}$, Pablo Avanzas ${ }^{5,6}$ and Daniel Hernández-Vaquero ${ }^{5,6 t}$ \\ ${ }^{1}$ Hospital Universitario de Canarias, Servicio de Cardiología, Tenerife, Spain, ${ }^{2}$ Departamento de Enfermería, Facultad de \\ Ciencias de la Salud, Universidad de La Laguna Tenerife, San Cristóbal de La Laguna, Spain, ${ }^{3}$ Departamento de Fisiología, \\ Facultad de Medicina, Universidad de La Laguna, Tenerife, San Cristóbal de La Laguna, Spain, ${ }^{4}$ Department of Cell Systems \\ and Anatomy UT Health San Antonio, Long School of Medicine, San Antonio, TX, United States, ${ }^{5}$ Área del Corazón, Hospital \\ Universitario Central de Asturias, Oviedo, Spain, ${ }^{6}$ Instituto de Investigación Sanitaria del Principado de Asturias, Oviedo, \\ Spain
}

Myocardial ischemia/reperfusion (IR) injury represents a critical problem associated with interventional approaches for coronary reperfusion. Pharmacological cardioprotective interventions are advocated to ameliorate IR injury. Melatonin is an anti-inflammatory and antioxidant agent with a wide range of therapeutic properties that may contribute to its cardioprotective effects. No systematic review or meta-analysis has compared melatonin vs. placebo as a cardioprotective agent in humans. The present study, based on a systematic review and meta-analysis, was carried out to assess melatonin's efficacy as a cardioprotective treatment. We performed a systematic review of the available literature. Randomized controlled trials (RCTs) were identified and information was extracted using predefined data extraction forms. The primary outcomes were (a) left ventricular ejection fraction (LVEF) and (b) blood troponin levels in patients who underwent myocardial revascularization and were randomized to melatonin or placebo. The inverse-variance random-effects method was used to pool the estimates. Heterogeneity and publication bias were assessed. Weighted mean differences or standardized mean differences were calculated. A total of 283 records were screened and seven RCTs met all the inclusion criteria. After the pooled analysis, the results on LVEF were consistent across all studies, and a significant heterogeneity was found in the results on troponin levels. The melatonin-treated patients had on average higher LVEF than the placebo-treated individuals with a weighted mean difference $=3.1 \%$ (95\% Cl 0.6-5.5, $p=0.01$ ). Five works compared the levels of troponin after melatonin or placebo treatment. The melatonin-treated patients had lower levels of troponin with a standardized mean difference $=-1.76(95 \% \mathrm{Cl}-2.85$ to $-0.67, p=0.002)$. The findings of this meta-analysis revealed that melatonin administration in humans as a cardioprotective agent attenuated heart dysfunction with a favorable effect on the LVEF.

Keywords: cardioprotection and ischemia-reperfusion injury, melatonin, meta-analysis, human, randomized controlled (clinical) trial 


\section{INTRODUCTION}

Based on epidemiological data, cardiovascular diseases are the number one cause of death globally, causing an estimated 17.9 million deaths each year (1). The most common form of cardiovascular disease, coronary artery disease, has seen a reduction in mortality over the past decades because of reperfusion medical strategies, such as coronary artery bypass grafting (CABG) and percutaneous coronary intervention (PCI). Although timely reperfusion has resulted in a substantial decline in mortality, significant morbidity remains with $22 \%$ heart failure at 1 year (2). This is, in part, due to the existence of myocardial ischemia/reperfusion (IR) injury, a phenomenon in which the restoration of coronary blood flows to ischemic myocardium results, paradoxically, in further myocardial injury and cardiomyocyte death (3).

The underlying mechanisms of myocardial IR injury include excessive reactive oxygen species generation, inflammation, and apoptosis. Great effort is needed to identify novel therapeutic strategies regulating myocardial oxidative stress and subsequent inflammation and apoptosis induced by myocardial IR injury (4). Despite numerous cardioprotective agents showing promising results in the experimental setting for preventing myocardial IR injury, their translation into the clinical setting for patient benefit has been challenging, and there is still no effective therapy (5).

Melatonin originally identified in the bovine pineal gland in 1958 (6) has multiple beneficial actions in various cardiac pathologies (7-10). Melatonin is a pleiotropic molecule with several functions that protect the heart against IR injury. It is well-established that melatonin possesses antioxidant and anti-inflammatory activities $(7,11)$. No systematic review or meta-analysis has been reported comparing melatonin and placebo as a cardioprotective agent in humans. The present study, based on a systematic review and metaanalysis, was carried out to assess melatonin's efficacy as a cardioprotective treatment.

\section{METHODS}

\section{Search Strategy}

We conducted and report this systematic review in accordance with the Preferred Reporting Items for Systematic Reviews and Meta-Analyses statement (12) and the Code of Ethics of the World Medical Association (Declaration of Helsinki). The consent of the patients was not required.

In October 2020, MEDLINE through PubMed, Embase, and the Cochrane Central Register of Controlled Trials were used to identify appropriate articles reporting the cardioprotection of melatonin in human myocardial IR injury. The full search strategy was as follows: [Melatonin (Title/Abstract)] AND [myocardial ischemia reperfusion injury (Title/Abstract) OR cardioprotection (Title/Abstract) OR heart disease (Title/Abstract) OR heart (Title/Abstract) OR acute coronary syndrome (Title/Abstract) OR acute myocardial infarction (Title/Abstract)]. Review articles, case report, and editorials were excluded.

\section{Inclusion and Exclusion Criteria}

Studies were eligible for inclusion if they met the following criteria: (a) melatonin compared with placebo; (b) reporting infarct size, expressed as the levels of troponin or left ventricular ejection fraction (LVEF) or the percentage of infarct area evaluated by magnetic resonance imaging; (c) human studies; and (d) randomized controlled trials (RCTs), compared with a control group. We excluded studies investigating the melatoninmediated cardioprotection in vitro or in vivo animal studies or publications not written in English.

The primary endpoint was to investigate the potential protective effect of melatonin against myocardial IR injury.

\section{Data Extraction and Evaluation of the Quality of the Studies}

Two members of the team (A.D-R. and P.A-G.) independently examined the title and abstract of the articles, selecting those publications that met the inclusion criteria. Two other members (N.B-F. and R.J.R.) evaluated the previously selected full-text articles and decided their final inclusion on an independent basis. These researchers were blinded to the names of the authors, the institutions, and the names of the journals. Disagreements were resolved through debate among these four team members. The quality of the studies was independently evaluated by two reviewers (P.A. and D.H-V.). To assess the methodological quality of the selected studies, the Cochrane Collaboration's table for assessing risk bias was used (13).

The data extracted from the selected studies were entered in an Excel spreadsheet. Two investigators (A.D-R. and N.BF.) independently extracted the data sets related to baseline information of the included studies (author, year, and sample size), reperfusion medical strategies (PCI or CABG), the methods for determining the infarct size (levels of troponin or LVEF or magnetic resonance imaging), and detailed therapeutic strategy (route of melatonin administration).

\section{Statistical Analysis}

The inverse-variance random-effects method was used to pool estimates. For LVEF, we used weighted mean differences. Because troponin was reported as I troponin or T troponin, for the metaanalysis of troponins, we used standardized mean differences (14). Heterogeneity was evaluated by Cochran's Q-test and by the calculation of $I^{2} . I^{2}<25 \%$ indicates low heterogeneity, $I^{2}$ $25-75 \%$ indicates moderate heterogeneity, and $>75 \%$ indicates high heterogeneity. For publication bias assessment, we used the Begg test and the Egger test. $p$-values $<0.05$ were considered statistically significant. All analyses were performed with the STATA v 16 (StataCorp, TX, USA).

\section{RESULTS}

\section{Selected Studies}

Figure 1 shows the study flowchart according to the PRISMA criteria. We evaluated 107 titles and abstracts; 82 were excluded and 25 articles required full-length article evaluation. Eighteen articles were excluded and only seven met all of the inclusion criteria and were included in the qualitative and quantitative 
pooled analyses (15-21). The total number of patients analyzed was 426. Patients' characteristics of each study are shown in Table 1.

\section{Effect of Melatonin Against Myocardial IR Injury Assessed by Blood Samples and Cardiac Imaging Troponin Levels}

Five RCTs (15-19) compared troponin levels between melatoninand placebo-treated individuals. Three $(15,17,19)$ of them reported levels of troponin I and two reported levels of troponin $\mathrm{T}(16,18)$. All works reported lower levels of troponin in the

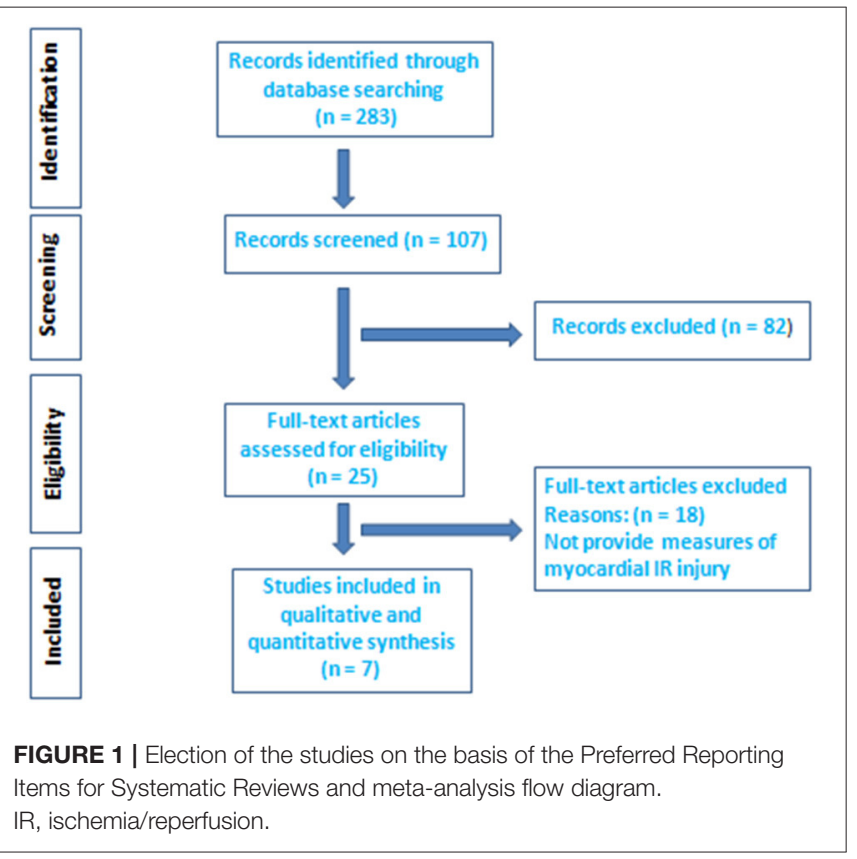

melatonin group. The standardized mean difference was -1.76 (95\% CI -2.85 to $-0.67, p=0.002$ ) (Figure 2). There was substantial heterogeneity among the studies; the $Q$ test had a $p$ value $<0.01$ and $I^{2}=79.8 \%$. There was no sign of publication bias; the Begg test had a $p$-value $=0.8$ and the Egger test had a $p$-value $=0.2$.

\section{LVEF}

Five studies (17-21) provided values of LVEF. Four studies found higher LVEF in the melatonin-treated patients. After the pooled analysis, the melatonin group had on average 3.1\% (95\% CI 0.65.5, $p=0.01$ ) higher LVEF than the placebo group (Figure 3). There was no sign of statistical heterogeneity; the $Q$ test had a $p$-value of 0.36 and $I^{2}=7.9 \%$. There was no sign of publication bias; the Begg test had $p=1$ and the Egger test had $p=0.24$.

\section{Magnetic Resonance Imaging}

Only two RCTs reported myocardial IR injury (infarct size), assessed by cardiac magnetic resonance imaging $(18,19)$. Due to the small number of studies, we did not perform a meta-analysis and only the systematic review is presented.

\section{DISCUSSION}

As far as we know, this is the first meta-analysis that provides a comprehensive evaluation of the possible benefits of melatonin in protecting the myocardium against IR damage in humans. To date, only seven RCTs (15-21) investigated melatonin as a therapeutic agent in the cardioprotective setting. Among them, three involved studies with myocardial infarction treated with PCI $(16,18,19)$ and four used CABG setting $(15,17,20,21)$.

In 2015, Ghaeli and colleagues (16) demonstrated that oral melatonin ( $3 \mathrm{mg}$ ) administered the night following PCI and continued daily during hospitalization had neutral effects on infarction size. The authors concluded that the study was underpowered and the dosing regimen was suboptimal. In 2017,

TABLE 1 | Overview of the studies included in the meta-analysis.

\begin{tabular}{|c|c|c|c|c|c|c|c|}
\hline References & $\begin{array}{l}\text { Methods for } \\
\text { determining the } \\
\text { infarct size }\end{array}$ & $\begin{array}{c}\text { Sample size } \\
\text { (n) }\end{array}$ & $\begin{array}{l}\text { Melatonin } \\
\text { administration }\end{array}$ & $\begin{array}{l}\text { Reperfusion } \\
\text { medical } \\
\text { strategies }\end{array}$ & Target disease & Effect & Risk of bias \\
\hline Gögenur et al. (15) & Levels of troponin I & 50 & $\begin{array}{l}\text { Melatonin orally and } \\
\text { intraoperatively }\end{array}$ & CABG & $\begin{array}{l}\text { Elective abdominal } \\
\text { aortic aneurysm } \\
\text { repair }\end{array}$ & $\mathrm{CP}$ & High \\
\hline Ghaeli et al. (16) & Levels of troponin $\mathrm{T}$ & 40 & Melatonin orally & $\mathrm{PCl}$ & AMI & No effect & High \\
\hline Dwaich et al. (17) & $\begin{array}{l}\text { LVEF, levels of } \\
\text { troponin I }\end{array}$ & 45 & Melatonin orally & CABG & CABG & $\mathrm{CP}$ & Low \\
\hline Ekeloef et al. (18) & $\begin{array}{l}\text { Levels of troponin T, } \\
\text { LVEF, and CRM }\end{array}$ & 48 & IV and IC melatonin & $\mathrm{PCl}$ & AMI & No effect & Low \\
\hline $\begin{array}{l}\text { Dominguez- } \\
\text { Rodriguez et al. } \\
\text { (19) }\end{array}$ & $\begin{array}{l}\text { Levels of troponin I, } \\
\text { LVEF, and CRM }\end{array}$ & 125 & IV and IC melatonin & $\mathrm{PCl}$ & AMI & $\begin{array}{l}\text { CP in the first } \\
\text { tertile (early after } \\
\text { symptom onset) }\end{array}$ & Low \\
\hline Shafiei et al. (20) & LVEF & 88 & Melatonin orally & CABG & CABG & $\mathrm{CP}$ & Low \\
\hline
\end{tabular}

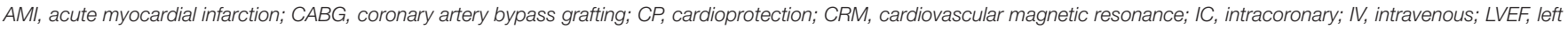
ventricular ejection fraction; PCl; percutaneous coronary intervention. 


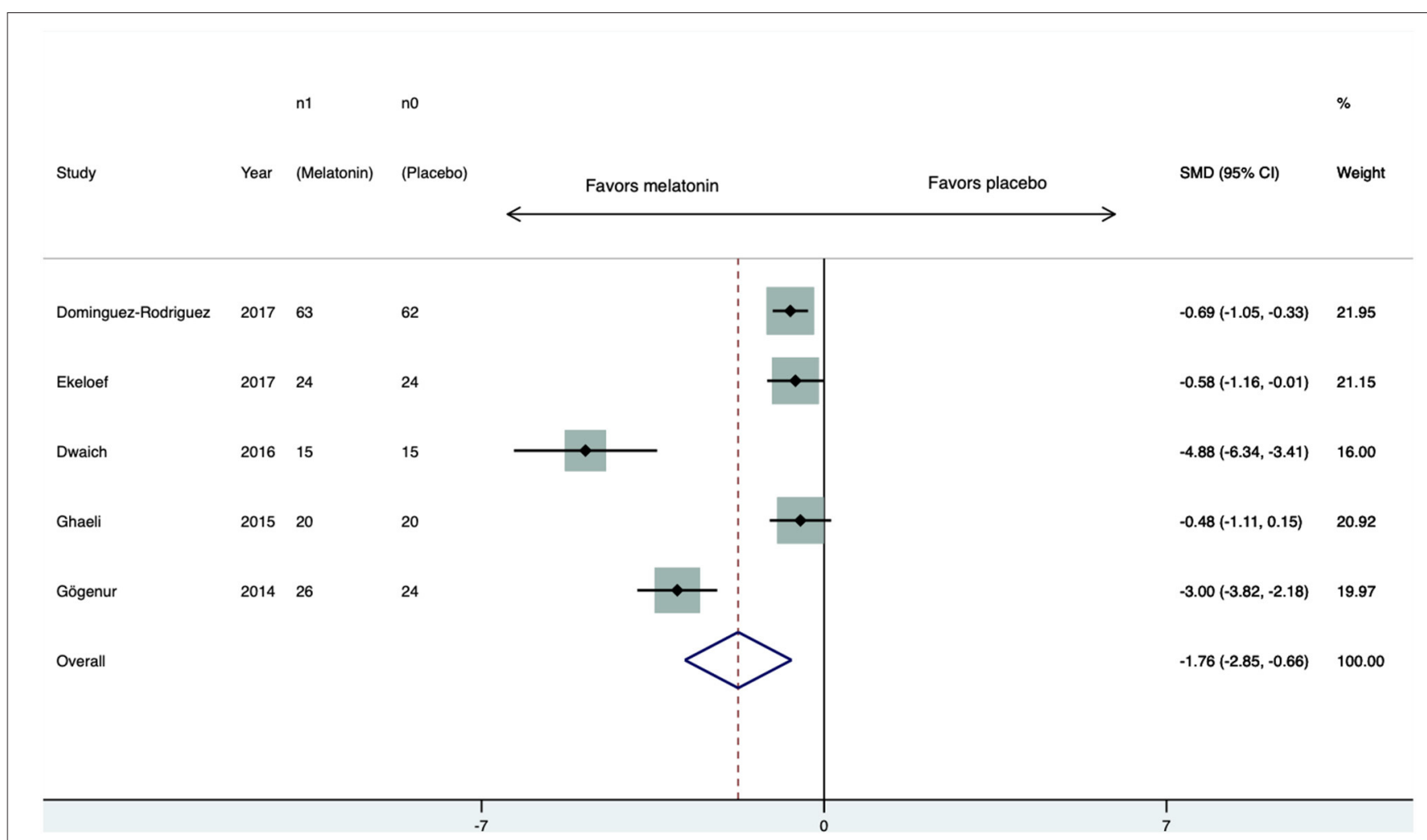

FIGURE 2 | Forest plot of troponin levels. Cl, confidence interval; SMD, standardized mean difference.

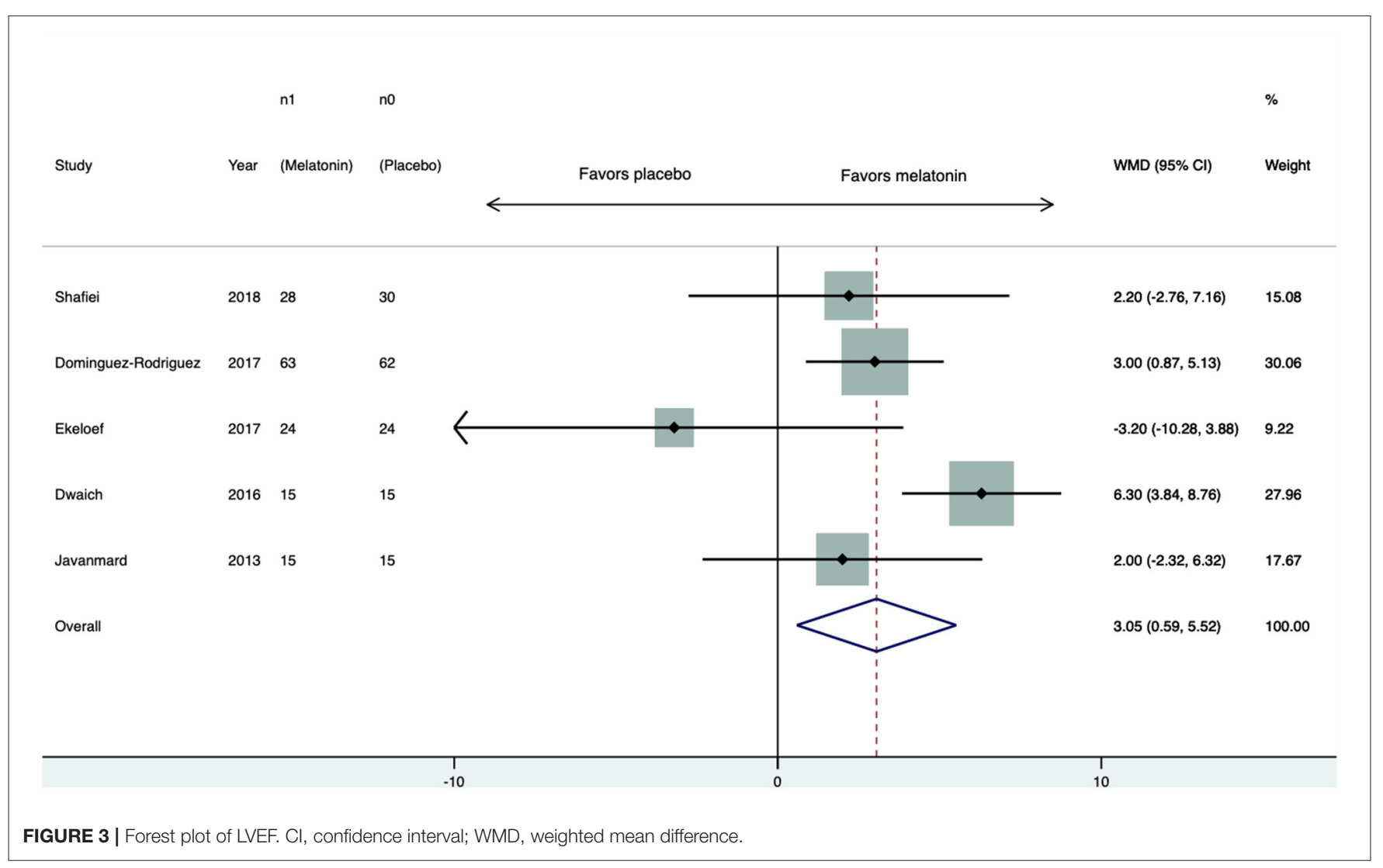


two studies provided additional data related to the effects of melatonin in patients with ST-elevation myocardial infarction $(18,19)$. Ekeloef and colleagues showed that $50 \mathrm{mg}$ intravenous and $1 \mathrm{mg}$ intracoronary melatonin at the onset of reperfusion failed to improve LVEF or reduce infarct size (18). A post hoc analysis of the MARIA trial demonstrated that early treatment (within $2 \mathrm{~h}$ ) with intravenous (12 $\mathrm{mg}$ ) and intracoronary (2 $\mathrm{mg}$ ) melatonin resulted in less infarct area in patients with STelevation myocardial infarction (19).

Others studies have reported cardioprotective effects using melatonin in patients undergoing CABG (15, 17, 20, 21). Gögenur et al. with a dose of $50 \mathrm{mg}$ melatonin given intraoperatively and $10 \mathrm{mg}$ melatonin given orally, during elective abdominal aortic aneurysm repair, demonstrated reduced post-operative troponin elevation and cardiac morbidity (15). A dose of 10 and $20 \mathrm{mg}$ of melatonin orally administered in patients who were undergoing elective CABG has previously been shown to reduce the degree of myocardial IR injury (17). Shafiei and colleagues demonstrated that $15 \mathrm{mg}$ of oral melatonin reduced CABG-related cardiac injury (20). Other authors have suggested that in patients who were undergoing elective CABG, $10 \mathrm{mg}$ of oral melatonin may be more effective as a cardioprotective agent when given prior to index ischemia (21).

Mao and colleagues (22) published a meta-analysis on this topic in animal studies. They demonstrated that pre-treatment with melatonin was associated with a significant lower infarct size in comparison with placebo in myocardial IR damage (weighted mean difference: $-20.45,95 \%$ CI -25.43 to $-15.47, p<0.001$; $\left.I^{2}=91.4 \%, p<0.001\right)$. Moreover, a similar improvement was also noted in LVEF, which indicated the role of melatonin in attenuating IR injury and subsequent cardiac dysfunction (22). Our meta-analysis showed a significant increase in LVEF for melatonin-treated patients in comparison with placebocontrolled individuals. In addition, there was a significant reduction in plasma levels of cardiac troponin as a result of melatonin treatment. While the results on LVEF were consistent across all studies, a significant heterogeneity was found in the results on troponin levels. Nevertheless, all works reported lower levels of troponin in melatonin-treated patients, and thus, the inconsistency only lies in how much troponin levels can be reduced using this drug.

Due to the small number of studies, we could not develop a subgroup analysis stratified by different doses of melatonin or according to its route of administration. The observed inconsistencies may be due to these different types of treatment since there is no consensus of the optimal clinical effective plasma concentration or drug administration $(7,23)$. Therefore, the timing and form of drug administration and approaches for infarct size determination may also have an impact on the outcomes.

Oxidative stress is the most established basic mechanism that causes myocardial tissue damage during reperfusion insult. It is characterized by severe imbalance between exaggerated reactive oxygen species generation and corresponding antioxidant defense systems (5). Numerous studies had verified the role of melatonin in alleviating IR injury in animals and explored the potential underlying mechanisms, which mainly focused on its powerful capacity to scavenge free radicals and activate antioxidant enzymes $(11,24-26)$. Speculatively, the cardioprotective effects of melatonin might be largely dependent on a clinically effective distribution of the drug in the myocardial area at risk, prior to ischemia and definitely prior to reperfusion.

This meta-analysis has limitations. First, the results of our meta-analysis are based on a small number of RCTs, uncertainty regarding the blinding of participants, and a small number of patients. Second, significantly high heterogeneity in the levels of cardiac troponin may affect the interpretation of the results. Third, due to the paucity of studies available, we found a moderate degree of inconsistency; therefore, we could not perform subgroup analysis or metaregression that could shed light in the presence of inconsistency. Finally, there was an obvious weakness in the published works regarding the evaluation of infarct size. Only two RCTs (18, 19) assessed myocardial IR injury site using cardiac magnetic resonance imaging; magnetic resonance imaging is considered the gold standard for the assessment of myocardial infarction size (27).

\section{CONCLUSION}

This meta-analysis revealed that melatonin administration in humans as a cardioprotective agent attenuated heart dysfunction with a favorable effect on the LVEF.

\section{DATA AVAILABILITY STATEMENT}

The original contributions presented in the study are included in the article/supplementary material, further inquiries can be directed to the corresponding author/s.

\section{AUTHOR CONTRIBUTIONS}

AD-R had full access to all of the data in the study and took responsibility for the integrity of the data and the accuracy of the data analysis. AD-R and PA-G: concept and design. NB-F, PA, and $\mathrm{DH}-\mathrm{V}$ : acquisition, analysis, or interpretation of data. AD-R and $\mathrm{DH}-\mathrm{V}$ : drafting of the manuscript. PA-G and RR: critical revision of the manuscript for important intellectual content. $\mathrm{DH}-\mathrm{V}$ : statistical analysis. AD-R: obtained funding. All authors contributed to the article and approved the submitted version.

\section{FUNDING}

This research was funded by Fundación Canaria Instituto de Investigación Sanitaria de Canarias (FIISC) (PIFUN11/18) and Sociedad Española de Cardiologia (SEC 2018 grant). 


\section{REFERENCES}

1. Timmis A, Townsend N, Gale CP, Torbica A, Lettino M, Petersen SE, et al. European Society of Cardiology: cardiovascular Disease Statistics 2019. Eur Heart J. (2020) 41:12-85. doi: 10.1093/eurheartj/ehz859

2. Niccoli G, Montone RA, Ibanez B, Thiele H, Crea F, Heusch G, et al. Optimized treatment of ST-elevation myocardial infarction. Circ Res. (2019) 125:245-58. doi: 10.1161/CIRCRESAHA.119.315344

3. Dominguez-Rodriguez A, Abreu-Gonzalez P, Reiter RJ. Cardioprotection and pharmacological therapies in acute myocardial infarction: challenges in the current era. World J Cardiol. (2014) 6:100-6. doi: 10.4330/wjc.v6.i3.100

4. Davidson SM, Ferdinandy P, Andreadou I, Bøtker HE, Heusch G, Ibáñez $\mathrm{B}$, et al. Multitarget strategies to reduce myocardial ischemia/reperfusion injury: JACC review topic of the week. J Am Coll Cardiol. (2019) 73:8999. doi: 10.1016/j.jacc.2018.09.086

5. Heusch G. Myocardial ischaemia-reperfusion injury and cardioprotection in perspective. Nat Rev Cardiol. (2020) 17:77389. doi: 10.1038/s41569-020-0403-y

6. Lerner $\mathrm{AB}$, Case JD, Takahashi Y. Isolation of melatonin and 5methoxyindole-3-acetic acid from bovine pineal glands. J Biol Chem. (1960) 235:1992-7. doi: 10.1016/S0021-9258(18)69351-2

7. Dominguez-Rodriguez A, Abreu-Gonzalez P, Chen Y. Cardioprotection and effects of melatonin administration on cardiac ischemia reperfusion: insight from clinical studies. Melatonin Res. (2019) 2:100-5. doi: 10.32794/mr11250024

8. Dominguez-Rodriguez A, Abreu-Gonzalez P, Avanzas P. The role of melatonin in acute myocardial infarction. Front Biosci (Landmark Ed). (2012) 17:2433-41. doi: $10.2741 / 4063$

9. Jiki Z, Lecour S, Nduhirabandi F. Cardiovascular benefits of dietary melatonin: a myth or a reality? Front Physiol. (2018) 9:528. doi: 10.3389/fphys.2018.00528

10. Fu Z, Jiao Y, Wang J, Zhang Y, Shen M, Reiter RJ, et al. Cardioprotective role of melatonin in acute myocardial infarction. Front Physiol. (2020) 11:366. doi: 10.3389/fphys.2020.00366

11. Dominguez-Rodriguez A, Abreu-Gonzalez P, Sanchez-Sanchez JJ, Kaski JC, Reiter RJ. Melatonin and circadian biology in human cardiovascular disease. $J$ Pineal Res. (2010) 49:14-22. doi: 10.1111/j.1600-079X.2010.00773.x

12. Moher D, Liberati A, Tetzlaff J, Altman DG, PRISMA Group. Preferred reporting items for systematic reviews and meta-analyses: the PRISMA statement. BMJ. (2009) 339:b2535. doi: 10.1136/bmj.b2535

13. Higgins JP, Altman DG, Gøtzsche PC, Jüni P, Moher D, Oxman AD, et al. The Cochrane Collaboration's tool for assessing risk of bias in randomised trials. BMJ. (2011) 343:d5928. doi: 10.1136/bmj.d5928

14. Higgins JP, Thompson SG, Deeks JJ, Altman DG. Measuring inconsistency in meta-analyses. BMJ. (2003) 327:557-60. doi: $10.1136 /$ bmj.327.74 14.557

15. Gögenur I, Kücükakin B, Panduro Jensen L, Reiter RJ, Rosenberg J. Melatonin reduces cardiac morbidity and markers of myocardial ischemia after elective abdominal aortic aneurism repair: a randomized, placebo-controlled, clinical trial. J Pineal Res. (2014) 57:10-5. doi: 10.1111/jpi.12138

16. Ghaeli P, Vejdani S, Ariamanesh A, HajhosseinTalasaz A. Effect of melatonin on cardiac injury after primary percutaneous coronary intervention: a randomized controlled trial. Iran J Pharm Res. (2015) 14:851-5.

17. Dwaich KH, Al-Amran FG, Al-Sheibani BI, Al-Aubaidy HA. Melatonin effects on myocardial ischemia-reperfusion injury: impact on the outcome in patients undergoing coronary artery bypass grafting surgery. Int J Cardiol. (2016) 221:977-86. doi: 10.1016/j.ijcard.2016.07.108

18. Ekeloef S, Halladin N, Fonnes S, Jensen SE, Zaremba T, Rosenberg J, et al. Effect of intracoronary and intravenous melatonin on myocardial salvage index in patients with ST-elevation myocardial infarction: a randomized placebo controlled trial. J Cardiovasc Transl Res. (2017) 10:4709. doi: 10.1007/s12265-017-9768-7

19. Dominguez-Rodriguez A, Abreu-Gonzalez P, de la Torre-Hernandez JM, Consuegra-Sanchez L, Piccolo R, Gonzalez-Gonzalez J, et al. Usefulness of early treatment with melatonin to reduce infarct size in patients with ST-segment elevation myocardial infarction receiving percutaneous coronary intervention (from the melatonin adjunct in the acute myocardial infarction treated with angioplasty trial). Am J Cardiol. (2017) 120:5226. doi: 10.1016/j.amjcard.2017.05.018

20. Shafiei E, Bahtoei M, Raj P, Ostovar A, Iranpour D, Akbarzadeh S, et al. Effects of $\mathrm{N}$-acetyl cysteine and melatonin on early reperfusion injury in patients undergoing coronary artery bypass grafting: a randomized, open-labeled, placebo-controlled trial. Medicine (Baltimore). (2018) 97:e11383. doi: 10.1097/MD.0000000000011383

21. Haghjooy Javanmard S, Ziaei A, Ziaei S, Ziaei E, Mirmohammad-Sadeghi M. The effect of preoperative melatonin on nuclear erythroid 2-related factor 2 activation in patients undergoing coronary artery bypass grafting surgery. Oxid Med Cell Longev. (2013) 2013:676829. doi: 10.1155/2013/676829

22. Mao ZJ, Lin H, Xiao FY, Huang ZQ, Chen YH. Melatonin against myocardial ischemia-reperfusion injury: a meta-analysis and mechanism insight from animal studies. Oxid Med Cell Longev. (2020) 2020:1241065. doi: 10.1155/2020/1241065

23. Andersen LP, Gögenur I, Rosenberg J, Reiter RJ. Pharmacokinetics of melatonin: the missing link in clinical efficacy? Clin Pharmacokinet. (2016) 55:1027-30. doi: 10.1007/s40262-016-0386-3

24. Dominguez-Rodriguez A, Abreu-Gonzalez P, Garcia-Gonzalez MJ, SamimiFard S, Reiter RJ, Kaski JC. Association of ischemia-modified albumin and melatonin in patients with ST-elevation myocardial infarction. Atherosclerosis. (2008) 199:73-8. doi: 10.1016/j.atherosclerosis.2007.10.019

25. Dominguez-Rodriguez A. Melatonin in cardiovascular disease. Expert Opin Investig Drugs. (2012) 21:1593-6. doi: 10.1517/13543784.2012.716037

26. Reiter RJ, Ma Q, Sharma R. Melatonin in mitochondria: mitigating clear and present dangers. Physiology (Bethesda). (2020) 35:85-96. doi: 10.1152/physiol.00034.2019

27. Ibanez B, Aletras AH, Arai AE, Arheden H, Bax J, Berry C, et al. Cardiac MRI endpoints in myocardial infarction experimental and clinical trials: JACC scientific expert panel. J Am Coll Cardiol. (2019) 74:238-56. doi: 10.1016/j.jacc.2019.05.024

Conflict of Interest: The authors declare that the research was conducted in the absence of any commercial or financial relationships that could be construed as a potential conflict of interest.

Copyright (C) 2021 Dominguez-Rodríguez, Abreu-González, Báez-Ferrer, Reiter, Avanzas and Hernández-Vaquero. This is an open-access article distributed under the terms of the Creative Commons Attribution License (CC BY). The use, distribution or reproduction in other forums is permitted, provided the original author(s) and the copyright owner(s) are credited and that the original publication in this journal is cited, in accordance with accepted academic practice. No use, distribution or reproduction is permitted which does not comply with these terms. 